\title{
Commentary
}

\section{Lasmiditan for the acute treatment of migraine: Subgroup analyses by prior response to triptans}

\author{
Fabíola Dach Éckeli \\ Faculdade de Medicina de Ribeirão Preto, USP, Ribeirão Preto, São Paulo, Brazil
}

$\bigotimes_{\text {fabioladach@usp.br }}$

Edited by

Marcelo Moraes Valença
The use of Lasmiditan, a highly selective agonist for the 5-HT1F receptor with penetration into the central nervous system, had its effectiveness proven for the acute treatment of migraine in two phase 3 studies, called SAMURAI and SPARTAN. In both studies, the percentage of patients who were pain-free after 2 $h$ in lasmiditan groups was higher than in the placebo group., ${ }^{2,3}$

Bearing in mind that lasmiditan and tryptans are different in structure and pharmacologically, the study in question aimed to investigate the response of lasmiditan for the acute treatment of migraine in relation to the previous response to tryptans (good or insufficient) and in those who never use this class of drug, based on the SAMURAI and SPARTAN studies.

Regarding the response to the acute treatment of migraine with lasmiditan in those who have already used triptan, lasmiditan proved to be effective both in those with good response to triptan and in those with insufficient response in the following outcomes: pain-free in $2 \mathrm{~h}$, absence of most uncomfortable symptoms in 2 hours and pain relief in 2 hours. The benefit was greater than placebo at all doses tested (50, 100 and 200 mg lasmiditan).

In those, who never used triptans, lasmiditan was effective in the acute treatment of migraine in doses of 100 and $200 \mathrm{mg}$ when compared with placebo for the same outcomes reported above. Taking into account that lasmiditan has no vasoconstrictor effects in animal models and in human, the results of the current study suggest that lasmiditan, the first tested drug of the new therapeutic class called "ditan", is a therapeutic option for patients with or without previous experience with triptans, as well as those with contraindications to that class of medication.

Fabíola Dach Éckeli

https://orcid.org/0000-0003-4249-4179

\section{Reference}

1. Knievel K, Buchanan AS, Lombard L, et al. Lasmiditan for the acute treatment of migraine: Subgroup analyses by prior response to triptans. Cephalalgia 2020; 40(1):19-27. doi: 10.1177/0333102419889350.

2. Kuca B, Silberstein SD, Wietecha L, et al. COL MIG-301 Study Group. Lasmiditan is an effective acute treatment for migraine: A phase 3 randomized study. Neurology 2018; 91:e2222-e2232.

3. Goadsby PJ, Wietecha LA, Dennehy EB, et al. Phase 3 randomized, placebocontrolled, double-blind study of lasmiditan for acute treatment of migraine. Brain 2019; 142:1894-1904. 